\title{
Nonlinear oscillation of a dielectric elastomer balloon
}

\author{
Jian Zhu, Shengqiang Cai and Zhigang Suo ${ }^{\text {a }}$ \\ School of Engineering and Applied Sciences, Harvard University, Cambridge, MA 02138
}

\begin{abstract}
This paper studies the dynamic behavior of a dielectric elastomer balloon subject to a combination of pressure and voltage. When the pressure and voltage are static, the balloon may reach a state of equilibrium. We determine the stability of the state of equilibrium, and calculate the natural frequency of the small-amplitude oscillation around the state of equilibrium. When the voltage is sinusoidal, the balloon resonates at multiple frequencies of excitation, giving rise to superharmonic, harmonic, and subharmonic responses. When the frequency of excitation varies continuously, the oscillating amplitude of the balloon may jump, exhibiting hysteresis.
\end{abstract}

a email: suo@seas.harvard.edu 


\section{Introduction}

Subject to a voltage through its thickness, a thin membrane of a dielectric elastomer reduces the thickness and expands the area. The strain of the membrane induced by the voltage can be large, readily exceeding 100\%. This and related phenomena are being developed for applications as electromechanical transducers. ${ }^{1-7}$ Much of the existing literature on dielectric elastomers has focused on quasi-static deformation, where the effect of inertia is negligible. In some of the potential applications, however, the elastomer deforms at high frequencies, up to 50 $\mathrm{kHz}$, and functions as vibration sources ${ }^{8}$, high-speed pumps ${ }^{9-12}$, and acoustic equipment ${ }^{13-14}$.

In a large-strain, high-frequency application, the elastomer may undergo nonlinear oscillation. While nonlinear oscillation has been studied in many areas of science and engineering, ${ }^{15,16}$ we are unaware of any theoretical analysis of nonlinear oscillation of dielectric elastomers. To explore the subject, this paper studies an idealized system: a dielectric elastomer balloon of a spherical shape, subject to a combination of pressure and voltage. Dielectric elastomer balloons have been studied as pumps ${ }^{12}$ and loudspeakers ${ }^{13}$, as well as an element of a shell-like actuator. ${ }^{17,18}$

The plan of this paper is as follows. Section 2 derives the equation of motion by using the method of virtual work. Section 3 describes the state of equilibrium when the pressure and voltage are static. Section 4 studies the small-amplitude oscillation around the state of equilibrium, and discusses the stability of the state of equilibrium against small perturbation. Section 5 studies parametric excitation, where the pressure is static but the voltage is sinusoidal. Section 6 shows that, when the frequency of excitation is varied continuously, the oscillating amplitude of the balloon can jump, exhibiting hysteresis.

\section{Equation of motion}


Fig. 1 illustrates a spherical balloon, radius $R$ and thickness $H$ in the undeformed state. The membrane of the balloon is a dielectric elastomer, taken to be incompressible, of density $\rho$. The membrane is coated on both faces with compliant electrodes. We will neglect any stress in the electrodes, but may add the mass of the electrodes to that of the membrane.

When the pressure inside the balloon exceeds the pressure outside by $p$ and the two electrodes are subject to a voltage $\Phi$, the balloon deforms to radius $r$ and the two electrode gain charges $+Q$ and $-Q$. Let $\lambda$ be the stretch of the membrane, namely,

$$
\lambda=\frac{r}{R}
$$

Let $D$ be the electric displacement in the membrane, namely,

$$
D=\frac{Q}{4 \pi r^{2}}
$$

The balloon is taken to deform under an isothermal condition, and the fixed temperature will not be considered explicitly. Consequently, the balloon is a thermodynamic system of two independent variables, $\lambda$ and $D$. We next formulate a model to evolve this system in time $t$.

The thermodynamics of the dielectric elastomer is characterized by the density of the Helmholtz free energy as a function of the two independent variables, $W(\lambda, D)$. We will adopt the model of ideal dielectric elastomer. ${ }^{19}$ The model assumes that the elastomer is a network of long and flexible polymers with a low density of crosslinks, so that the crosslinks almost do not constrain the process of polarization. Once the effect of crosslinks on polarization is neglected, the dielectric behavior of the elastomer is liquid-like, unaffected by deformation. Consequently, the free-energy function of the dielectric elastomer is written as a sum of two parts:

$$
W(\lambda, D)=\frac{\mu}{2}\left(2 \lambda^{2}+\lambda^{-4}-3\right)+\frac{D^{2}}{2 \varepsilon}
$$

The first part is the elastic energy, where $\mu$ is the shear modulus. For simplicity, we use the neo-Hookean model to describe the elasticity of the network; other models of elasticity may be 
used. The second part in (3) is the dielectric energy, where $\varepsilon$ is the permittivity. For an ideal dielectric elastomer, the permittivity is taken to be a constant independent of deformation.

The model of ideal dielectric elastomers has been used almost exclusively in the literature to analyze devices. However, recent experiments on VHB, the most studied dielectric elastomer, have shown that the permittivity may vary by a factor of 2 when the elastomer undergoes large deformation. ${ }^{18}$ See [20] for a review of thermodynamic models for dielectric elastomers. While the general considerations in this paper are valid for arbitrary function $W(\lambda, D)$, we will use the function (3) in numerical calculations.

When the charge on the electrodes varies by $\delta Q$, the applied voltage does work $\Phi \delta Q$. When the radius of the balloon varies by $\delta r$, the pressure does work $4 \pi r^{2} p \delta r$, and the inertial force does work $-4 \pi R^{2} H \rho\left(d^{2} r / d t^{2}\right) \delta r$. We neglect any viscous force. Thermodynamics dictates that, for arbitrary variation of the system, the variation of the free energy of the membrane should equal the work done by the voltage, the pressure, and the inertia, namely,

$$
4 \pi R^{2} H \delta W=\Phi \delta Q+4 \pi r^{2} p \delta r-4 \pi R^{2} H \rho \frac{d^{2} r}{d t^{2}} \delta r
$$

Inserting (1) and (2) into (4), and recalling that the balloon is a system of two independent variables, $\lambda$ and $D$, we obtain that

$$
\begin{gathered}
\frac{\partial W(\lambda, D)}{\partial \lambda}=\frac{2 \Phi D \lambda}{H}+\frac{p R}{H} \lambda^{2}-R^{2} \rho \frac{d^{2} \lambda}{d t^{2}} \\
\frac{\partial W(\lambda, D)}{\partial D}=\frac{\Phi}{H} \lambda^{2}
\end{gathered}
$$

Equation (5) balances momentum, and (6) enforces electrostatic equilibrium. For an ideal dielectric elastomer, (6) recovers the liquid-like dielectric behavior, $D=\varepsilon E$, where $E$ is the electric field.

Inserting (3) into (5) and (6), and eliminating $D$, we obtain that 


$$
\frac{d^{2} \lambda}{d t^{2}}+g(\lambda, p, \Phi)=0
$$

with

$$
g(\lambda, p, \Phi)=2 \lambda-2 \lambda^{-5}-p \lambda^{2}-2 \Phi^{2} \lambda^{3}
$$

Eq. (7) is the equation of motion that evolves the stretch of the balloon as a function of time, $\lambda(t)$. This equation is consistent with that derived in Ref. [12]. In writing (7) and (8), we have normalized the time by $R \sqrt{\rho / \mu}$, the pressure by $\mu H / R$, and the voltage by $H \sqrt{\mu / \varepsilon}$.

\section{A balloon in a state of equilibrium under static pressure and voltage}

When the two loading parameters, $p$ and $\Phi$, are both static, the balloon may reach a state of equilibrium, of stretch $\lambda_{e q}$. In the state of equilibrium, the equation of motion, (7), reduces to

$$
g\left(\lambda_{e q}, p, \Phi\right)=0
$$

This nonlinear algebraic equation determines $\lambda_{e q}$ for given values of $p$ and $\Phi$.

Fig. 2 plots (9) as pressure-stretch curves at several values of voltage. When $\Phi=0$, the problem reduces to that of a pressurized balloon, a well known problem in the literature of nonlinear elasticity. ${ }^{21}$ As the balloon expands, the pressure first increases, reaches a peak, and then decreases. The peak pressure corresponds to a critical state. When the applied pressure is above the peak, the balloon cannot reach a state of equilibrium. When the applied pressure is below the peak, corresponding to each value of the pressure are two values of the stretch. The value of the stretch on the rising part of the curve corresponds to a stable state of equilibrium, and the value of the stretch on the descending part of the curve corresponds to a state of unstable equilibrium.

When $\Phi \neq 0$, charges of opposite signs are induced on the two electrodes. The attraction between the electrodes causes the membrane of the balloon to reduce thickness and increase 
area. As shown in Fig. 2, the voltage lowers the critical pressure, and increases the stretch of any stable state of equilibrium.

Fig. 3 plots the condition of equilibrium (9) as voltage-stretch curves at several values of pressure. When $p=0$, as the balloon expands, the voltage first increases, reaches a peak, and then decreases. This behavior is understood as follows. ${ }^{12,}{ }^{22-26}$ The voltage induces a positive charge on one electrode, and negative charge on the other electrode. The oppositely charged electrodes attract each other, so that the dielectric membrane reduces its thickness. For a prescribed voltage, the reduction in the thickness of the membrane increases the electric field. This positive feedback leads to electromechanical instability, or pull-in instability. The peak of the curve corresponds to the critical state. The critical voltage is reduced in the presence of the pressure.

\section{Small-amplitude oscillation around a state of equilibrium}

Consider a state of equilibrium, $\lambda_{e q}$. When the balloon is perturbed from this state of equilibrium, we write

$$
\lambda(t)=\lambda_{e q}+\Delta(t)
$$

where $\Delta(t)$ is the amplitude of perturbation, and is taken to be small. We then expand the function $g(\lambda, p, \Phi)$ as a power series in $\Delta$ around the equilibrium stretch $\lambda_{e q}$. Consequently, to the leading order in $\Delta$, the equation of motion (7) becomes

$$
\frac{d^{2} \Delta}{d t^{2}}+\Delta \frac{\partial g}{\partial \lambda}\left(\lambda_{e q}, p, \Phi\right)=0
$$

where the partial derivative

$$
\frac{\partial g(\lambda, p, \Phi)}{\partial \lambda}=2+10 \lambda^{-6}-2 p \lambda-6 \Phi^{2} \lambda^{2}
$$


is evaluated at $\lambda=\lambda_{\text {eq }}$, and is a constant independent of time. We may call this partial derivative the stiffness of the balloon.

When the stiffness is negative, the perturbation $\Delta(t)$ will grow exponentially in time, and the state of equilibrium is unstable. When the stiffness is positive, the balloon will oscillate around the state of equilibrium, and the state of equilibrium is stable. Inspecting (11), we see that the natural frequency $\omega_{0}$ of the small-amplitude oscillation around the stable state of equilibrium is determined by

$$
\omega_{0}^{2}=\frac{\partial g}{\partial \lambda}\left(\lambda_{e q}, p, \Phi\right)
$$

When $p$ and $\Phi$ are prescribed at static values, the equilibrium stretch $\lambda_{e q}$ is determined by (9), and the natural frequency $\omega_{\mathrm{o}}$ is determined by (13). To avoid solving the nonlinear algebraic equation (9), we rearrange (9) and (13) to express $p$ and $\Phi$ in terms of $\lambda_{e q}$ and $\omega$, namely,

$$
\begin{gathered}
\Phi^{2}=-\lambda_{e q}^{-2}+7 \lambda_{e q}^{-8}-\frac{1}{2} \lambda_{e q}^{-2} \omega_{0}^{2}, \\
p=4 \lambda_{e q}^{-1}-16 \lambda_{e q}^{-7}+\lambda_{e q}^{-1} \omega_{0}^{2}
\end{gathered}
$$

By varying $\lambda_{\text {eq }}$, we plot this pair of equations in Fig. 4 as curves of constant $\omega_{0}$ on the plane of $(p, \Phi)$. Each point on the curve labeled by $\omega_{0}=0$ corresponds to a critical state of the balloon. The coordinates of the point gives the critical values of $p$ and $\Phi$. When the pressure and voltage fall above this curve, the balloon cannot reach a state of equilibrium. When the pressure and voltage fall below the curve, the balloon can reach a stable state of equilibrium, and the natural frequency of the small-amplitude oscillation around the state of equilibrium can be read from the diagram. As indicated in Fig.4, the frequency is normalized by a group of parameters, $R^{-1} \sqrt{\mu / \rho}$. These parameters are fixed for a given balloon. However, a change in the static pressure or the static voltage will tune the natural fervency. 


\section{Parametric excitation}

When the pressure or the voltage varies with time, the dynamic behavior of the balloon can be very complex. To illustrate the complexity, we prescribe a static pressure $p$ and a sinusoidal voltage:

$$
\Phi(t)=\Phi_{d c}+\Phi_{a c} \sin \Omega t
$$

where $\Phi_{d c}$ is the dc voltage, $\Phi_{a c}$ is the amplitude of the ac voltage, and $\Omega$ is the frequency of excitation. The equation of motion (7) becomes

$$
\frac{d^{2} \lambda}{d t^{2}}+2 \lambda-2 \lambda^{-5}-p \lambda^{2}-2\left(\Phi_{d c}+\Phi_{a c} \sin \Omega t\right)^{2} \lambda^{3}=0
$$

The oscillatory voltage is a source of energy, and appears in a coefficient of the ordinary differential equation (17). Phenomena of this type are known as parametric excitation..$^{15,16}$

The time-dependent stretch $\lambda(t)$ can be obtained by solving (17) for any initial conditions. We set $p R / \mu H=0.1$ and $\varepsilon \Phi_{d c}^{2} / \mu H^{2}=0.1$. Were these values of pressure and voltage static, the balloon could attain a state of equilibrium $\lambda_{e q}=1.029$, or oscillate around the state of equilibrium at the natural frequency $\omega_{0} R \sqrt{\rho / \mu}=3.096$. We use this state of equilibrium as the initial conditions in our numerical simulations. We then apply the oscillatory voltage with specific values of $\Phi_{a c}$ and $\Omega$. Once the numerical solution of $\lambda(t)$ attains a steady state of oscillation, we define the amplitude of oscillation as the half of the difference between the maximal and minimal values of the stretch.

Fig. 5 plots the amplitude of oscillation as a function of the frequency of excitation. The balloon resonates most strongly when the frequency of excitation is around the natural frequency, $\Omega \approx \omega_{0}$. The balloon also resonates when the frequency of excitation is several times the natural frequency, for example, $\Omega \approx 2 \omega_{0}$, a response is known as subharmonic resonance. ${ }^{16}$ In addition, the balloon resonates when the frequency of excitation is a fraction of the natural 
frequency, for example, $\Omega \approx \omega_{0} / 2$, a response known as superharmonic resonance. Resonance at multiple values of the frequency of excitation is common for parametric excitation.27,28

Fig. 6 shows the numerical results of $\lambda(t)$ for three values of the frequency of excitation, $\Omega \approx \omega_{0} / 2,, \omega_{0}, 2 \omega_{0}$. Independent of the frequency of excitation $\Omega$, the balloon always oscillates near the natural frequency $\omega_{0}$, as defined by the small-amplitude oscillation around a state of equilibrium.

Experimental data reported in the literature show that the frequency of the oscillation doubles, or is close to, that of the ac voltage. ${ }^{10,11}$ For example, in figure 70 of $[11], \Omega \approx \omega_{0} / 2$, while in figure 72 in [11], $\Omega \approx \omega_{0}$. Our numerical results not only show superharmonic and harmonic resonance, but also show subharmonic resonance with $\Omega \approx 2 \omega_{0}$. This discrepancy between the theory and the experiment need be resolved in future studies.

\section{Jump in oscillating amplitude when the frequency of excitation varies}

To obtain other essential characteristics of the nonlinear oscillation, we study equation (17) by using the method of harmonic balance ${ }^{15,16}$. The time-dependent stretch is approximated as

$$
\lambda(t)=\lambda_{e q}+a(t) \cos \omega t+b(t) \sin \omega t
$$

where $\lambda_{e q}$ is the stretch in the state of equilibrium, $a$ and $b$ are time-dependent amplitudes, and $\omega$ is the frequency of oscillation. We assume that the amplitudes vary slowly with time. We use the truncated Fourier series, and neglect terms of high-frequencies, $2 \omega, 3 \omega$, etc.

We are interested in the harmonic oscillation in a steady state, where $a$ and $b$ are constants, and the balloon oscillates at the frequency equal to the frequency of excitation, $\omega=\Omega$. With the method of harmonic balance, we substitute (18) into (17), set the coefficients of the constant, $\cos \Omega t$ and $\sin \Omega t$ to be zero, and neglect terms of higher frequencies. Then we obtain 
three nonlinear equations for $\lambda_{e q}, a$ and $b$. We solve these nonlinear equations for $a$ and $b$ by using the Newton-Raphson method. Fig.7 plots the oscillating amplitude of the balloon, $\sqrt{a^{2}+b^{2}}$, as a function of the frequency of excitation $\Omega$. Upon increasing the frequency of excitation, the steady-state solution will start from point $\mathrm{O}$, to A, to $\mathrm{D}$, then jump to point $\mathrm{E}$, then to F, and to O'. However, upon decreasing the frequency of excitation, the steady-state will start from point O', to F, to E and C. Similar phenomena of hysteresis have been reported in many parametrically excited oscillators..$^{16,29}$

The above interpretations are better understood when we study how the amplitudes vary with time, $a(t)$ and $b(t)$. Since $a$ and $b$ are taken to vary with time slowly, $d^{2} a / d t^{2}$ and $d^{2} b / d t^{2}$ are neglected. Taking second derivative of (18) with respect to $t$, we obtain that

$$
\frac{d^{2} \lambda}{d t^{2}}=\left(-\omega^{2} a+2 \omega \frac{d b}{d t}\right) \cos \omega t+\left(-2 \omega \frac{d a}{d t}-\omega^{2} b\right) \sin \omega t
$$

Substituting (19) into (17), setting $\Omega=\omega$, and equating the coefficients of $\cos (\Omega t)$ and $\sin (\Omega t$ ) to zero, we obtain that

$$
\frac{d a}{d t}=F(a, b), \quad \frac{d b}{d t}=G(a, b) .
$$

The two functions $F(a, b)$ and $G(a, b)$ are lengthy and are not listed here. Given initial values $a(0)$ and $b(\mathrm{o})$, we can evolve (20) to obtain $a(t)$ and $b(t)$.

Fig. 8 shows the phase plane of $(a, b)$. Steady-state solution 1, related to point $A$ in Fig. 7, is a center point. Steady-state solution 2, related to point $B$ in Fig. 7, is a saddle point. Steadystate solution 3, related to point $C$ in Fig. 7, is a center point. Fig. 8 shows that the parametric response depends on the initial condition. For example, if the initial condition is close to the center point $1, a(0)=0$ and $b(0)=0.1$, the final path will cycle around this center point. If the initial condition is close to the saddle point 2, however, the final path will not stay near the saddle point, but will follow either a larger cycle, say path (1), or a small cycle, say path (2). 
The stability analysis of steady-state solution is as follows. With the infinitesimal perturbation for the steady-state solution, let

$$
a(t)=a^{o}+\alpha(t), \quad b(t)=b^{o}+\beta(t),
$$

where $a^{\circ}$ and $b^{\circ}$ are the steady-state solution, and $\alpha(t)$ and $\beta(t)$ are infinitesimal perturbation. Substituting (21) into (20), keeping the linear term for $\alpha$ and $\beta$, we obtain

$$
\left[\begin{array}{l}
\frac{d \alpha}{d t} \\
\frac{d \beta}{d t}
\end{array}\right]=\left[\begin{array}{ll}
\frac{\partial F}{\partial a} & \frac{\partial F}{\partial b} \\
\frac{\partial G}{\partial a} & \frac{\partial G}{\partial b}
\end{array}\right]\left[\begin{array}{l}
\alpha \\
\beta
\end{array}\right]
$$

The partial derivatives of the functions $F(a, b)$ and $G(a, b)$ are evaluated at the steady-state solution $\left(a^{\mathrm{o}}, b^{\mathrm{o}}\right)$. The steady-state solution is stable when the eigenvalues of the matrix in (22) have negative real parts. This analysis produces results indicated on the phase plane.

\section{Concluding remarks}

We describe a dielectric elastomer balloon with an equation of motion of one degree of freedom. When the pressure and the voltage are static, the balloon may reach a state of equilibrium. We study the stability of the state of equilibrium against small perturbation, and calculate the natural frequency of the small-amplitude oscillation around the state of equilibrium. The natural frequency is tunable by varying the pressure or the voltage. When the pressure is static but the voltage is sinusoidal, the balloon resonates at multiple values of the frequency of excitation, giving rise to superharmonic, harmonic and subharmonic responses. Furthermore, when the frequency of excitation is changed continuously, the oscillating amplitude of the balloon may jump at certain values of the frequency of excitation, exhibiting hysteresis. We hope to further analyze the nonlinear dynamic behavior by using models of many degrees of freedom, including dissipation due to viscosity and leakage. We also hope that future experiments will ascertain the theoretical predictions. 


\section{References}

1. Pelrine R, Kornbluh R, Pei QB and Joseph J, Science 287: 836-839 (2000).

2. $\quad$ Plante JS and Dubowsky S, Int. J. Solids Struct. 43: 7727-7751 (2006).

3. $\quad$ Wissler M and Mazza E, Sens. Actuators A 134: 494-504 (2007).

4. Kofod G, Wirges W, Paajanen M and Bauer S, Appl. Phys. Lett. 9o, o81916 (2007).

5. $\quad$ Patrick L, Gabor K and Silvain M, Sens. Actuators A 135: 748-757 (2007).

6. Carpi F, Rossi DDe, Kornbluh R, Pelrine R and Sommer-Larsen P, Dielectric Elastomers as Electromechanical Transducers, Elsevier, Oxford (2008).

7. Carpi F., Frediani G. and Rossi DDe, IEEE/ASME Trans. Mechatronics. Hydrostatically coupled dielectric elastomer actuators. In press (2009).

8. Bonwit N, Heim J, Rosenthal M, Duncheon C and Beavers A, Proceedings of SPIE 6168: $39-48$ (2006).

9. Fox JW and Goulbourne NC, J. Mech. Phys. Solids 56: 2669-2686 (2008).

10. Fox JW and Goulbourne NC, J. Mech. Phys. Solids (2009), doi:10.1016/j.jmps.2009.03.008.

11. Fox JW, Electromechanical Characterization of the Static and Dynamic Response of Dielectric Elastomer Membranes, Master thesis, Virginia Polytechnic Institute and State University (2007).

12. Mockensturm EM and Goulbourne NC, Int. J. Non-Linear Mech. 41: 388-395 (2006).

13. Chiba S, Waki M, Kornbluh R and Pelrine R, Proceedings of the SPIE 6524: 652424 (2007).

14. Heydt R, Kornbluh R, Eckerle J and Pelrine R, Proceedings of SPIE 6168: 61681M (2006).

15. Jordan DW and Smith P, Nonlinear Ordinary Differential Equations, Clarendon Press, Oxford (1987).

16. Nayfeh AH and Mook DT, Nonlinear Oscillation, Wiley, New York (1979). 
17. Lochmatter P, Development of a shell-like electroactive polymer (EAP) actuator, ETH dissertation (2007).

18. Wissler M and Mazza E, Sens. Actuators A 138: $384-393$ (2007).

19. Zhao X, Hong W and Suo Z, Phys. Rev. B 76: 134113 (2007).

20. Zhao X and Suo Z, J. Appl. Phys. 104: 123530 (2008).

21. Holzapfel GA, Nonlinear Solid Mechanics, John Wiley \& Sons, New York (2000).

22. Stark K H and Garton CG, Nature 176: 1225-1226 (1955).

23. Zhao X and Suo Z, Appl. Phys. Lett. 91: 061921 (2007).

24. $\quad$ Norris AN, Appl. Phys. Lett. 92: 026101 (2008)

25. Liu YJ, Liu LW, Zhang Z, Shi L and Leng JS, Appl. Phys. Lett. 93: 106101 (2008).

26. $\quad$ Diaz-Calleja R, Riande E and Sanchis MJ, Appl. Phys. Lett. 93: 101902 (2008).

27. Turner KL, Miller SA, Hartwell PG, MacDonald NC, Strogatz SH and Adams SG, Nature 396: 149-152 (1998).

28. $\quad$ De SK and Aluru NR, Phys. Rev. Lett. 94: 204101 (2005).

29. Lifshitz R and Cross MC, Phys. Rev. B 67: 134302 (2003). 


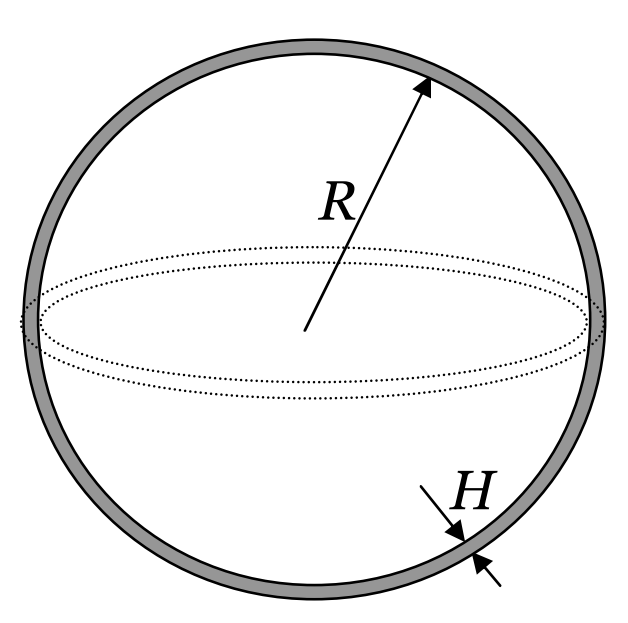

Reference State

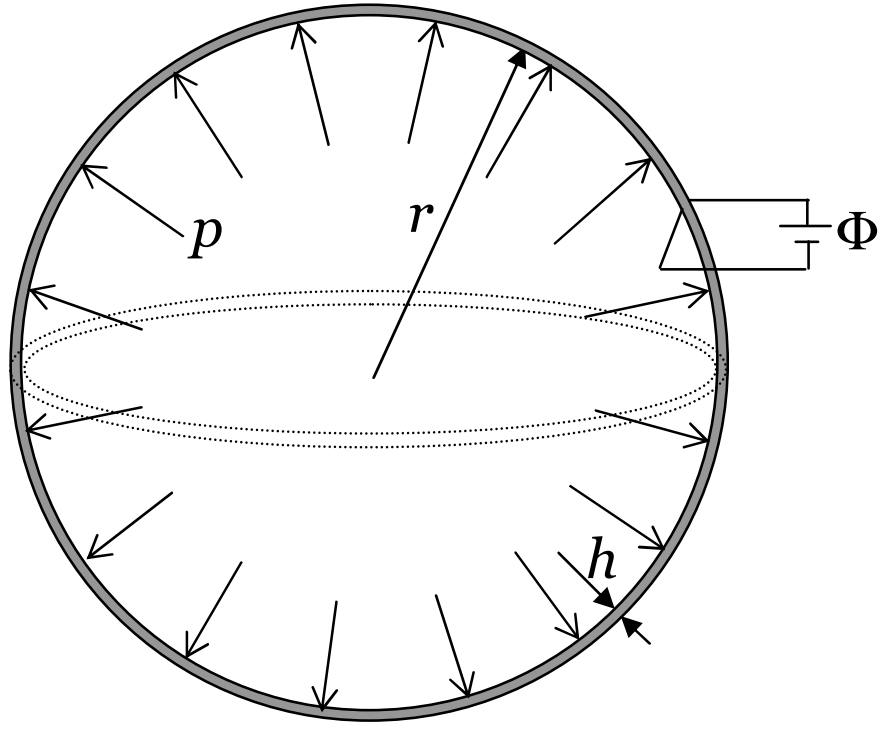

Current State

Fig. 1. An dielectric elastomer balloon deforms under a pressure and a voltage. 


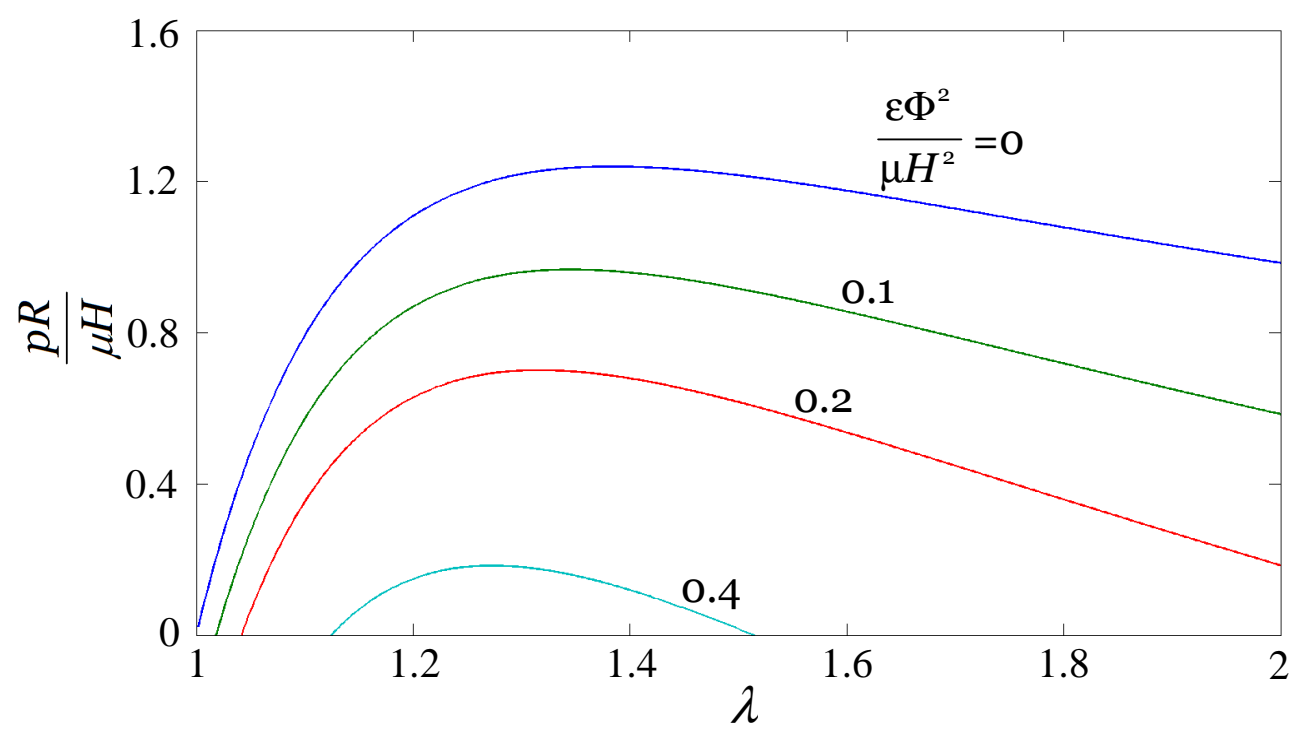

Fig. 2. Subject to a static pressure and voltage, the balloon may reach a state of equilibrium. The pressure is plotted as a function of the equilibrium stretch at several values of the voltage. 


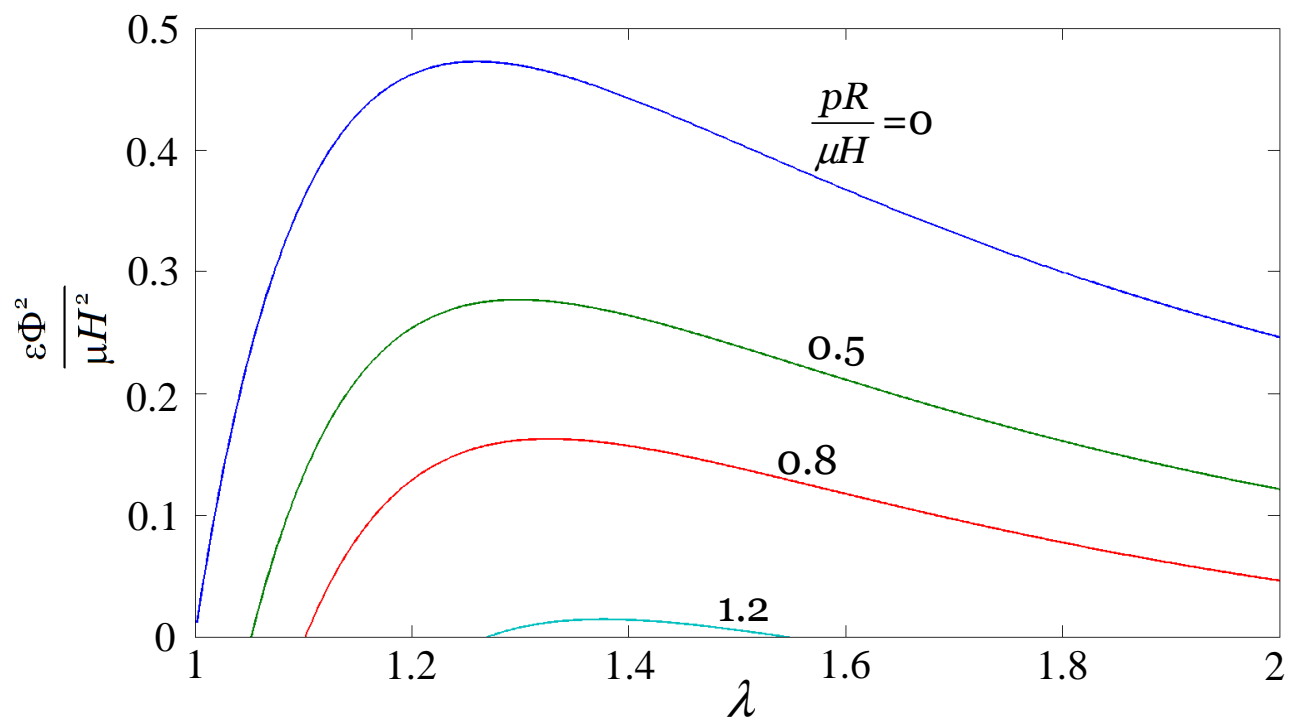

Fig. 3. The voltage is plotted as a function of the equilibrium stretch at several values of the pressure. 


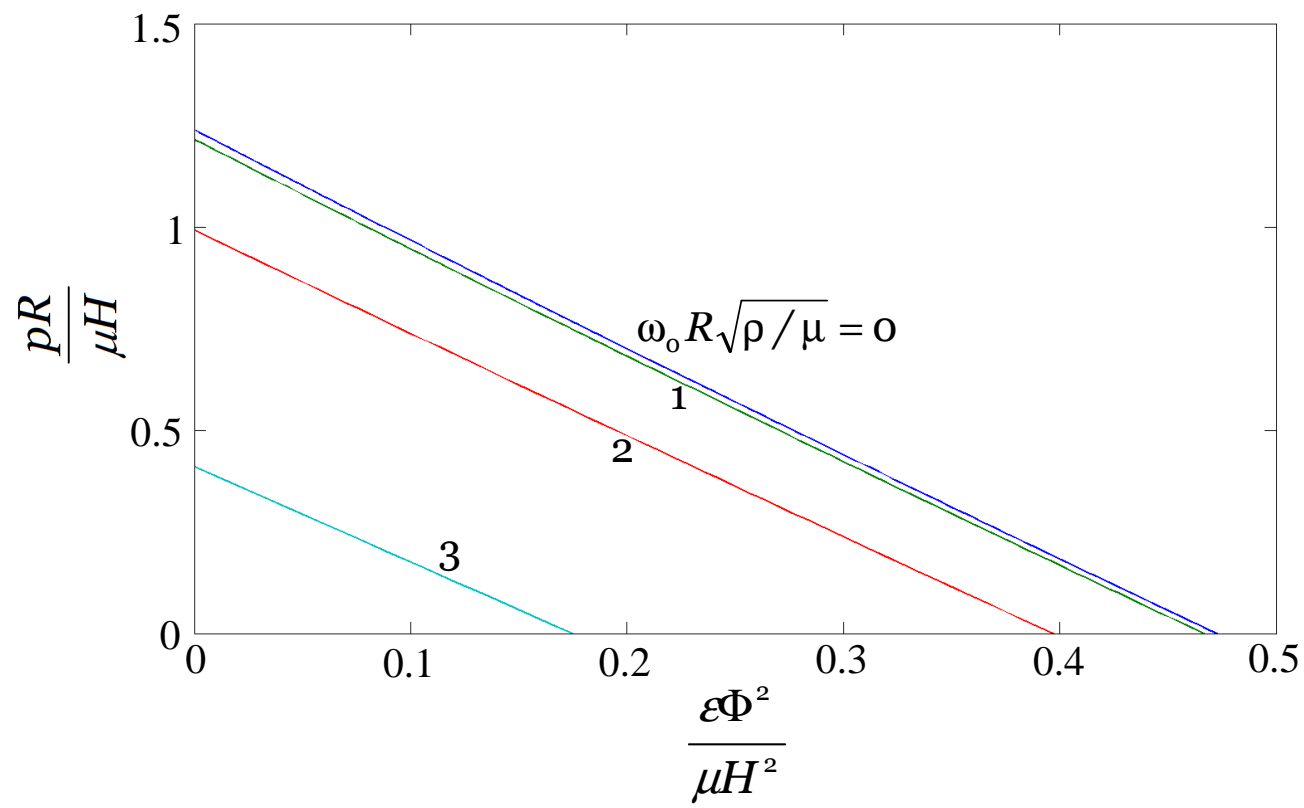

Fig.4. Plotted on the plane of $(p, \Phi)$ are curves of constant natural frequency. The curve labeled by $\omega_{0}=0$ corresponds to the critical conditions. Above this curve, the balloon cannot reach a state of equilibrium. Below the curve, the balloon can reach a stable state of equilibrium. In this region, each point $(p, \Phi)$ corresponds to a state of stable equilibrium, and the balloon can oscillate around the state at the natural frequency $\omega_{0}$. 

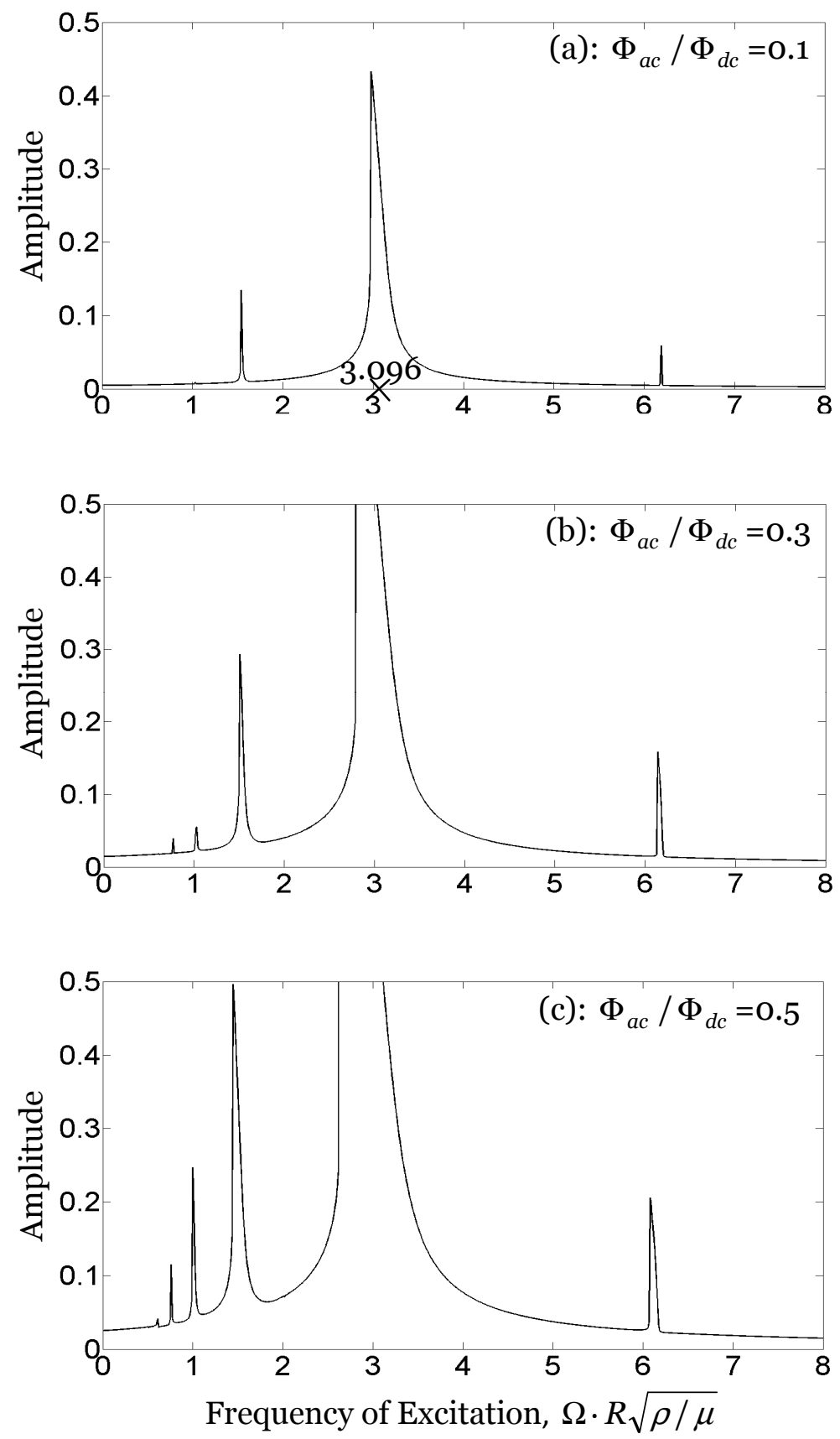

Fig.5. Excited by a sinusoidal voltage, the balloon resonates at several values of the frequency of excitation $\Omega$. The oscillating amplitude of the balloon is plotted as a function of the frequency of excitation for $p R / \mu H=0.1$ and $\varepsilon \Phi_{d c}^{2} / \mu H^{2}=0.1$, at selected values of $\Phi_{a c} / \Phi_{d c}$. 

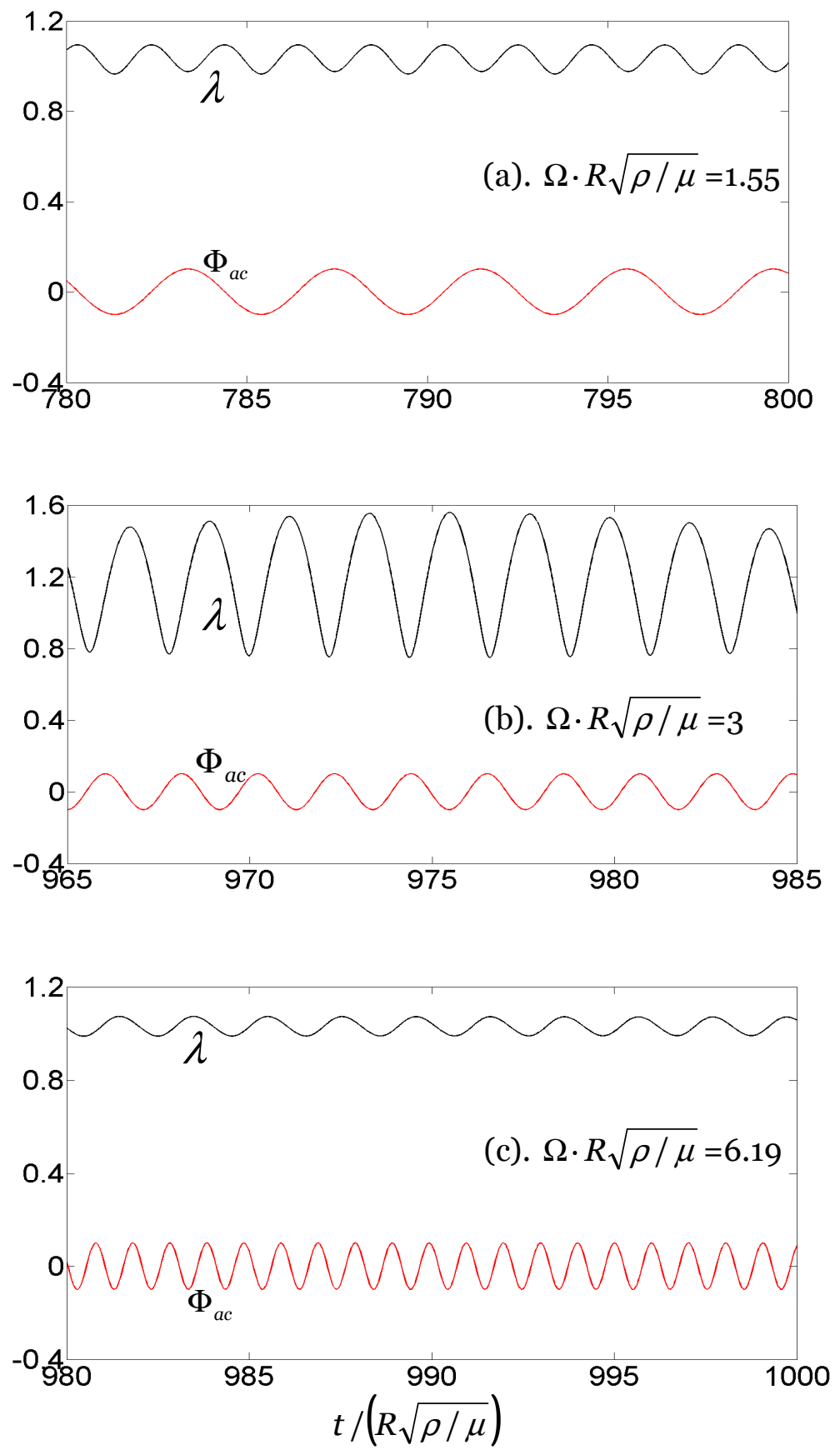

Fig.6. Superharmonic, harmonic, and subharmonic responses $\left(p R / \mu H=0.1, \varepsilon \Phi_{d c}^{2} / \mu H^{2}=0.1\right.$, and $\left.\Phi_{a c} / \Phi_{d c}=0.1\right)$ 


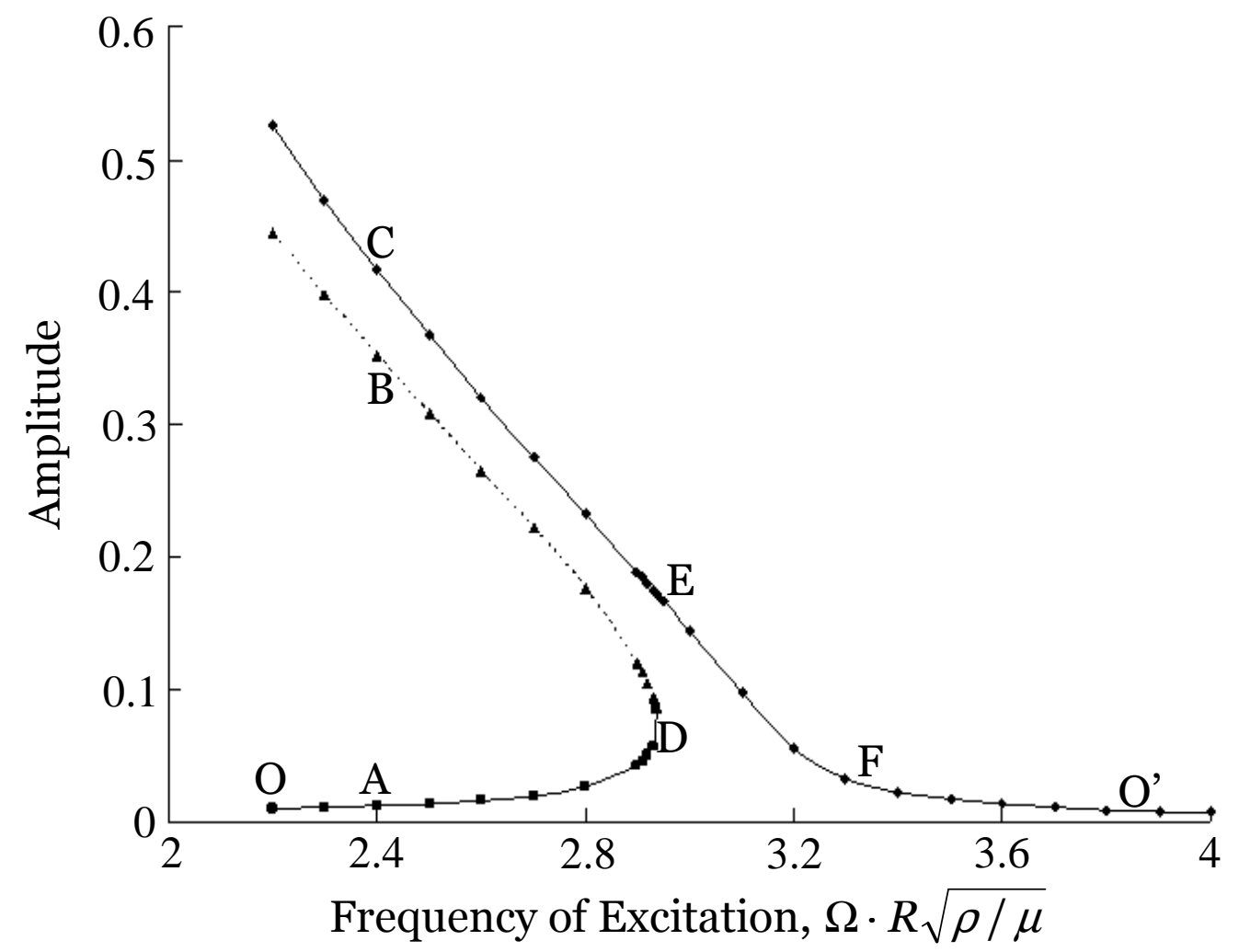

Fig. 7. Steady-state solutions when $p R / \mu H=0.1, \varepsilon \Phi_{d c}^{2} / \mu H^{2}=0.1$, and $\Phi_{a c} / \Phi_{d c}=0.1$ 


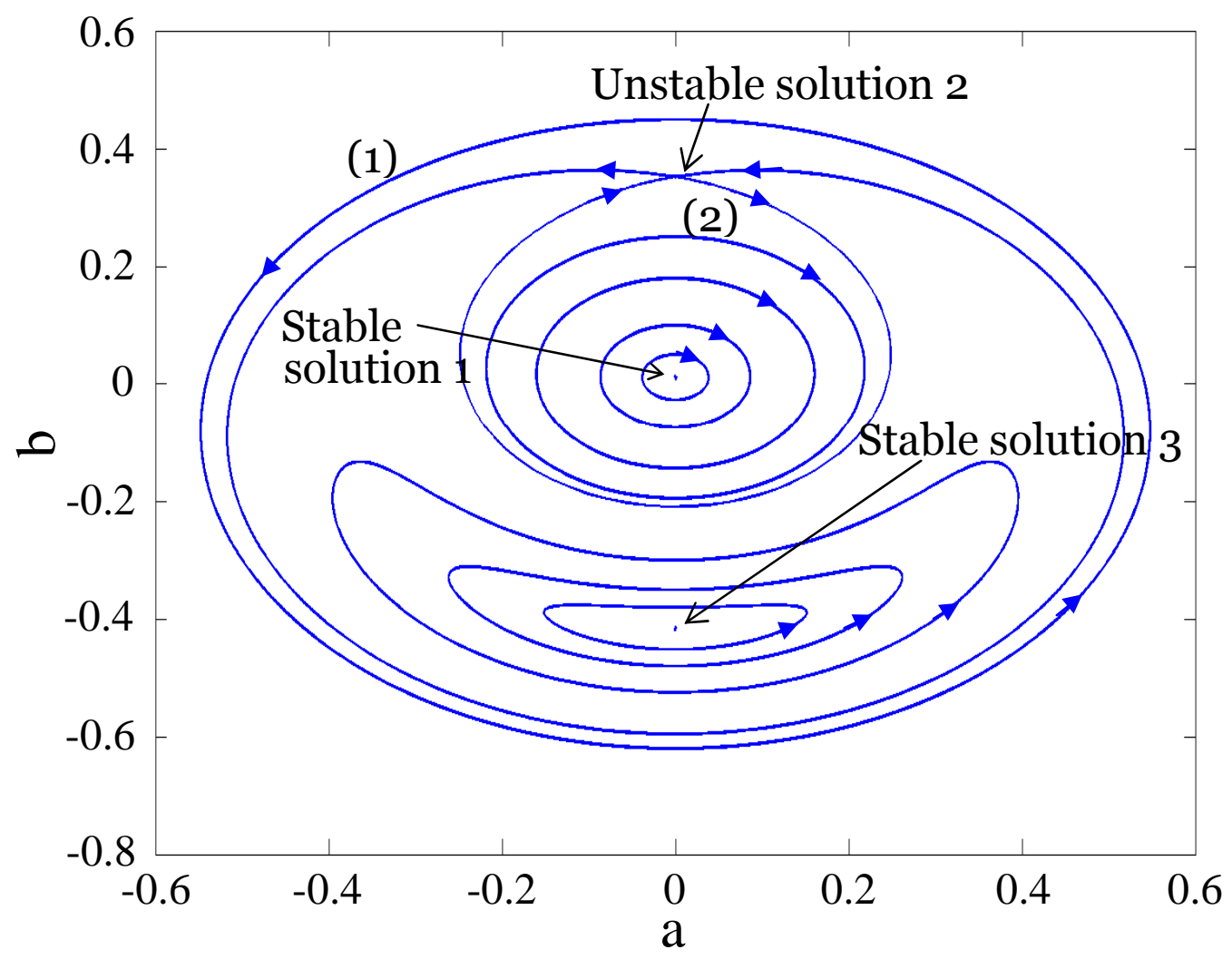

Fig. 8 Phase plane of $a$ and $b$ when $\Omega \cdot R \sqrt{\rho / \mu}=2.4, p R / \mu H=0.1, \varepsilon \Phi_{d c}^{2} / \mu H^{2}=0.1$, and $\Phi_{a c} / \Phi_{d c}=0.1$ 